\title{
Zielvorgaben verfehlen das Ziel
}

\section{Urs Stoffel}

Dr. med., Mitglied des FMH-Zentralvorstandes, Departementsverantwortlicher Ambulante Versorgung und Tarife

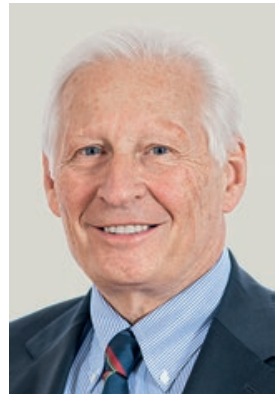

Man kann das «Ding» nennen, wie man will: «Zielvorgaben» oder «Kostenbremse» [1], letztlich ist und bleibt die Massnahme der «Zielvorgaben» ein verkapptes Globalbudget im ambulanten Bereich. Genau das sieht aber das KVG im ambulanten Bereich nicht vor. In einem von der FMH in Auftrag gegebenen Gutachten kommt Prof. Ueli Kieser zum Schluss, dass die Massnahmen einer Zielvorgabe nicht verfassungskonform sind. Denn das KVG ist als Versicherungsgesetz konzipiert. Versicherte haben laut KVG Anspruch auf die definierten Leistungen der OKP; dieser Anspruch kann nicht beschränkt werden, um damit die Kosten zu steuern und zu dämpfen. Wenn Sie analog eine Autoversicherung haben, so könnte die Versicherung auch nicht sagen, dass sie Ihren versicherten Schaden nicht übernimmt, weil Sie im laufenden Jahr bereits zu viele Autounfälle hatten.

Nachdem die Massnahme «Zielvorgaben» mittlerweile aus dem Kostendämpfungspaket 2 herausgelöst und zum indirekten Gegenvorschlag der KostenbremseInitiative erklärt wurde (siehe auch Seite 728: «Die erstaunliche Karriere der Zielvorgabe im Bundesrat»), muss diese Massnahme besonders genau unter die Lupe genommen werden. Gerade wenn sie als Gegenvorschlag zu einer Initiative tauglich sein soll. Eine solche Massnahme wird letztlich zulasten der Patientinnen und Patienten zur Sparmassnahme, weil sie die medizinische Versorgung und damit die Qualität verschlechtert. Man möchte also der Ärzteschaft hier die Aufgabe zuschieben, die Patientenversorgung nach politischen Kostenkriterien zu «steuern» und damit de facto einzuschränken.

Zielvorgaben sind nicht verfassungskonform, denn sie schränken den Leistungsanspruch der Versicherten ein, der ihnen laut KVG zusteht.

Eine Budgetvorgabe zieht zwangsläufig eine Begrenzung der Leistungen nach sich. Das führt wiederum zu Wartezeiten und durch die Bevorzugung der Privatzahler letztlich zu einer Zweiklassenmedizin. Der Bundesrat schreibt in seinen Erläuterungen, dass der Zugang zu den Versicherungsleistungen für die Bevölkerung in jedem Fall gewährleistet sei. Budgets sind aber nur dann kostensparend, wenn sie Leistungen einschränken, was schon aus mathematischen Über- legungen auf der Hand liegt. Wenn aber sämtliche versicherten Leistungen gewährleistet sein sollen, dann kann das Budget kaum Einsparungen bringen, wird aber zugleich sicher administrative Mehrkosten verursachen. Interessanterweise zeigen Länder mit Budgetvorgaben, dass die Kosten langfristig nicht weniger ansteigen. Die Daten der OECD-Länder belegen, dass in Prozentanteilen des BIP beispielsweise die Niederlande trotz Globalbudget keine Kostenvorteile aufweisen und verglichen mit der Schweiz sogar eine ungünstigere Kostenentwicklung zeigen.

Eine Zielvorgabe ist immanent mit einer Prognose verknüpft. Sie muss also vorhersagen können, wie sich der Bedarf für medizinische Leistungen in Zukunft entwickelt. Dieser Bedarf ist aber von sehr vielen komplexen Parametern abhängig. Bereits eine unterschätzte Grippewelle kann eine solche Zielvorgabe infrage stellen. Gerade in der jetzigen Pandemie werden wir uns dessen schmerzlich bewusst. Der Bund ist beispielsweise in den letzten Jahren wiederholt daran gescheitert, die Kostenentwicklung korrekt zu prognostizieren. Über Jahre hinweg lagen die Prämien höher als die Kosten der Grundversorgung [2]. Wären die Kostenprognosen des Bundes immer richtig, hätten wir wohl über Jahre hinweg zu wenig behandelt. Umgekehrt würden zu tief prognostizierte Gesundheitskosten dazu führen, dass für die medizinischen Behandlungen zu wenig Geld zur Verfügung stünde und die hohe Qualität der Gesundheitsversorgung in der Schweiz somit gefährdet würde.

In der zweiten Corona-Welle wollte sich der Bundesrat nicht einmal auf die Auslastung der Intensivstationen in den nächsten fünf Tagen festlegen [3]. Jetzt meint er aber, man könne die medizinisch notwendigen Gesundheitskosten des übernächsten Jahres - im Detail je Versorgungssektor und Kanton - korrekt vorhersagen. Wir bleiben dabei: Zielvorgaben im Gesundheitswesen sind ebenso wie die «Kostenbremse-Initiative» ein verkapptes Globalbudget und verfehlen das Ziel einer guten Patientenversorgung.

\section{Literatur}

1 www.nzz.ch/schweiz/wenn-das-volk-die-katze-im-sack-kauftld.1380117

2 www.derbund.ch/schweiz/standard/hoffnungen-auf-einen-milden-praemienherbst/story/15445438

3 saez.ch/article/doi/saez.2020.19390 